\title{
Psychiatric Nurses Explanations of Self-Harming Behaviour in Secure Forensic Settings: A Multi-Method Phenomenological Investigation
}

Peter T Sandy*

Principal Lecturer (Mental Health), Buckinghamshire New University, Uxbridge, London, UK

\begin{abstract}
Aim: This paper reports on a study that explores psychiatric nurses explanations of self-harming behaviors in secure forensic psychiatric environments.

Background: There is evidence suggesting that there is a differential perception between psychiatric nurses and service users of the reasons for self-harming behaviors in secure settings. This gulf in perception, which may negatively impact on the care offered to service users, is a function of nurses' lack of or limited knowledge of self-harm. Yet, research concerning nurses' reasons for service users' self-harming behaviors is limited.
\end{abstract}

Methods: The study utilized a phenomenological methodology with semi-structured individual interviews $(n=25)$ and focus group interviews $(n=6 \times 6)$. The data were analyzed thematically using Interpretative Phenomenological Analysis.

Results: The findings indicate that the behavior of self-harm does not have fixed causes rather it has multiple causes which, in the main, relate to affect regulation, limited coping skills, rigid institutional regime and practitioners ${ }^{*}$ negative attitudes.

Conclusions: These findings have implications for practice and recommendations are made to improve this. Regular training and support should be provided for psychiatric nurses in secure environments in order to broaden their understanding of self-harm and its underlying motives. Improved understanding of self-harm may result in improved nurse-service user relationships and thus, safer and effective care provision.

Keywords: Explanations; Phenomenology; Nurses; Secure environments; Self-harm

\section{Introduction}

A major problem facing healthcare professionals and researchers around the world in relation to self-harm is that its incidence is increasing [1]. Estimates of its incidence are noted to vary widely, with the United Kingdom (UK) having the highest in Europe [2]. This variation in rates is likely to be a function of lack of uniformity of definitions, reflected in the differential perceptions of healthcare professionals and researchers about the behaviour. Self-harm is defined, for the purpose of this paper, as self-injury, initiated by an individual, regardless of intent, which directly results in harm to any part of the body of that individual. This definition focuses on cutting, a behaviour commonly encountered in secure environments.

Secure hospitals are places with the highest incidence of self-injury in both female and male service users [3]. Most psychiatric nurses in these hospitals have little understanding of self-harm [4]. So, exposure to this behaviour, particularly when frequent, can generate intense stress in them, which in turn may impede the care offered to service users [5]. Yet, there is a paucity of literature that discusses nurses' knowledge of self-harm. This paper reports on a study that intends to help fill this gap by exploring psychiatric nurses' explanations of selfharming behaviours in secure settings.

\section{Background}

Self-harm is a growing problem in secure environments, as it is a behaviour that is often repeated by service users in these settings. Service users sometimes hide their injuries from healthcare professionals and even from their families [6]. When detected, they may provide excuses, misleading clinicians into believing, for example, that their injuries are the result of accidents or attacks from others [7]. Hence, it is difficult to accurately determine its incidence. Despite this, its growing rate is a significant indicator of the extent of the problem.
Self-harm is a multidimensional behaviour, and such complexity indicates a multitude of reasons motivating service users to hurt themselves. Tension release is one of the most commonly cited reasons by practitioners, as service users tend to claim experiences of reduced tension following acts of self-injury [8-10]. Service users who use self-harm for this reason have difficulties with verbal communication [11]. Self-harm is a potent medium for communicating and regulating emotions, such as anger. James and Warner [12] and Pembroke [13] agree with this and report that service users sometimes hurt their bodies to release frustration and as a form self-punishment or to avenge others.

Self-harm has a protective function for service users. Females who have been sexually abused sometimes use it to make their bodies look unattractive. Such unattractiveness, caused by the scars, protects them from unwanted sexual advances. Added to this, females with abusive experience have been reported in some studies to cut their bodies in order to clean themselves of aspects of abusers left inside them [14]. Such feelings of dirtiness may develop to feelings of self-hatred, selfblame and guilt. These emotions can be tormenting and service users may attempt to alleviate the same using approaches such as depersonalisation or dissociation [15,16]. Although dissociative feelings are protective in function in the context of not allowing individuals to

*Corresponding author: P. T. Sandy, Principal Lecturer, Buckinghamshire New University, Uxbridge Campus106 Oxford Road, Uxbridge, London, Middlesex UB8 1NA, United Kingdom, Tel: +44(0)1494522141 ext 4451; E-mail: Peters.sandy@bucks.ac.uk

Received August 01, 2012; Accepted August 27, 2012; Published August 30 2012

Citation: Sandy PT (2012) Psychiatric Nurses Explanations of Self-Harming Behaviour in Secure Forensic Settings: A Multi-Method Phenomenological Investigation. J Nurs Care 1:120. doi:10.4172/2167-1168.1000120

Copyright: (c) 2012 Sandy PT. This is an open-access article distributed under the terms of the Creative Commons Attribution License, which permits unrestricted use, distribution, and reproduction in any medium, provided the original author and source are credited. 
face their unbearable experiences, they can also in themselves be very frightening [17]. Self-mutilation enables people to return to reality which, in turn, ends episodes of distressing experiences.

Being locked up with many rules and limited freedom can also be very distressing for service users, particularly when they perceive their future to be bleak [18]. Users in secure settings usually perceive themselves to lack control and/ or be out of control of their lives $[11,19,20]$. Being out of control can be frightening for anyone, but it can be particularly worrying for individuals who self-harm. In secure environments, self-harm provides users a sense of having control of something or being in charge of their own lives [21,22]. However, people are generally disinclined to injure themselves because the behaviour is socially embarrassing and stigmatising [23]. But in secure settings where others self-injure, the inhibition against such behaviours is reduced. Service users who self-injure in these settings, in the main, receive solicitous attention. This makes this behaviour more attractive for those who look forward to similar support. Hence, self-harm is a common behaviour in secure settings, considered to be a learned behaviour often repeated by service users [24].

Service users are sometimes described by psychiatric nurses as attention seekers and manipulators [25]. While such a response is ethically questionable, it could enhance people's need to selfharm. Service users find these responses insulting and offensive, as they are absolutely different from their behavioural motives $[5,26]$. Such differential perceptions are the result of limited knowledge and understanding of the reasons for self-harm. This is of grave concern since service users are often distressed about their experiences, and depend on nurses to understand what is happening to them. Psychiatric nurses who cannot clearly articulate the reasons for self-harm are unlikely to establish the partnership necessary for effective care provision. Although innumerable publications are available on the subject of selfharm, only very few have explored healthcare professionals including nurses ' understanding of the same in secure hospitals. Consequently, self-harm remains a poorly understood behavioural phenomenon in these areas and therefore worthy of comprehensive investigation.

\section{Method}

The present study used interpretative phenomenological analysis (IPA), developed by Smith [27] to ensure in-depth exploration of people's personal experiences of phenomena. IPA recognises that the meanings people attribute to experiences can be understood in the socio-cultural contexts in which they are experienced [28]. This is consistent with this study, which seeks to develop knowledge about participants' understanding of self-harm in a specific context, secure forensic environments. Proponents of IPA claim that speech, and especially conversation, is the medium of all understanding, as it is through conversation that meanings are developed and understood [29]. Explanations for service users' self-harming behaviour were revealed in this study during participant-researcher interactions. IPA acknowledges that the meanings of phenomena can only be accessed through an interpretative process with researchers assuming both existential and transcendental positions in relation to issues examined [30]. This approach, which requires researchers to use their preconceptions to understand individuals' personal worlds and the meaning they attribute to them, was adopted in this study and enabled the researchers to develop comprehensive insights into self-harm.

The study site consisted of 15 locked clinical environments with about 22 nurses working in each area. The study adopted a two-stage approach to data collection. Stage one was the individual interviews and stage two was the focus group interviews. Sampling for both sets of interviews was purposive, as participants were selected on the basis of having experience with self-harm. The sample size for the individual interviews was 25 and that for the focus groups was 36 with six participants per group. Ethical approval was gained from the National Research Ethics Services (NRES) and Research site Ethics Committee (REC). Consent was sought and obtained from each participant before data collection.

Data were collected using a semi-structured interview guide consistent with IPA principles [27]. The data collection was commenced with individual interviews, followed by focus group interviews. Interviews lasted from 45 to 60 , and were commenced with explanations of the research purpose and ethical issues. All interviews were audio taped and contemporaneous notes were taken during the process.

The audio recorded interview data were transcribed verbatim and analysed using IPA guidelines [31]. Analysis proceeded in parallel with the interviews and was inductive. Analysis was conducted iteratively throughout the interview period until thematic category saturation was achieved. It was carried out individually by the researcher and themes generated were agreed through discussion.

\section{Results}

The narratives are noted to contain rich explanations of service users' self-harming behaviour in secure settings. A significant part of the explanations provided are the functions which self-harm serves in the lives of service users. They are presented here fewer than three key thematic categories: internally motivated functions, externally motivated functions and institutional issues. Each of these themes contains a number of sub-thematic categories. Extracts from participants ' narratives are used to support the discussions of identified themes. Data from focus group interviews are identified by the initials "Fg" and that from individual interviews by "In".

\section{Internally motivated functions}

A close examination of the transcripts revealed a wide range of purposes which self-harm may serve. Some of the purposes were generated from within service users and the most commonly cited among these was that of communication and regulation of distress.

\section{Communication and regulation of distress}

A commonly reported opinion of participants, irrespective of the methods of self-harm, was that this behaviour is mainly used to express feelings. It was asserted by some participants that service users often achieve emotional release and thus; feel calmer following acts of selfinjury. In relation to this, they repeatedly mentioned that service users tend to engage in self-harming acts only when their feelings become unbearable and unutterable.

Service users do it out of frustration when they can no longer cope with being locked up and cannot do what they would like to do (In).

When participant was prompted to expand on her meaning of frustration, she stated:

Frustration could come from service users not being allowed to go out on leave. Hurting themselves is a way of telling others about their level of unhappiness (In).

Some participants spoke of frustration as an outcome of an accumulation of angry feelings over a period of time. They claimed that some service users tend to bottle-up their distress and, on most occasions, use self-harm as a channel to release the same. Users who 
tend to do this generally lack the ability to verbally express their emotions.

A service user I nursed on this ward told me that she gets frustrated by the locked doors and many rules. She does not know other ways of dealing with her frustration (Fg).

Self-harm appears to be a function of limited or lack of other strategies to deal with anger. Although anger was the emotion most frequently mentioned by participants during interview encounters, only a minority claimed that it often precedes incidents of self-harm.

The service users of this unit sometimes get angry because of the way we treat them. Cutting is the best way to get their anger out (Fg).

\section{Cleansing}

Cutting was considered by participants the most common form of self-harm in secure settings. One obvious outcome of cutting reported was bleeding. For the most part, this was noted in participants narratives to be associated with the notion of cleansing; expulsion of "badness" or "dirtiness" from within.

Most of the service users who harm themselves have been sexually abused. These experiences make them feel dirty. Cutting themselves and letting the blood flow is self-cleansing. This gets the abuser out that is inside their body $(\mathrm{Fg})$.

\section{Prevention of abuse}

The accounts presented thus far indicate that people self-harm for several reasons. It was highlighted in few practitioners ' transcripts that the scars which subsequently develop when people wound themselves play significant roles in preventing future abuse.

Most people I cared for have been abused in the past. They cut themselves to look ugly. Ugliness drives abusers away. It makes them feel safe (Fg).

While this extract does explicitly indicate a positive aspect of scars, prevention of abuse, it covertly demonstrates participants ' perceptions of self-harm as a means for individuals to regain control over their body.

\section{Regaining control}

This theme focuses on participants ' perception of the relationship between self-harm and feelings of loss or lack of control. Two facets of control were revealed following a close examination of the narratives. These were control of external factors (such as the physical environment) and control of internal factors, which in essence refers to people's emotional states. Participants' discussions concentrated on the latter as they repeatedly made attempts to explain service users ' quest for assuming ownership of their bodies.

People who self-harm in this unit have been sexually abused. Hurting themselves distracts them from their distress, creates a feeling of being in control (Fg).

People who have been sexually abused tend to experience thoughts of powerlessness and loss of control to change their circumstances. The experience of such an emotional state, as reported by some participants, is related to service users' self-image and subsequent self-harming behaviours.

One service user told me that whenever he cuts himself, other thoughts, such as being nasty inside go away and he feels in charge of his body (Fg).

In contrast to this way of coping, participants indicated that service users sometimes try to manage their traumatic experiences by detaching themselves from the situations. Events of dissociation were described by a minority of participants mainly in terms of service users ' inability to feel emotionally or physically. They stressed that the latter, physical numbness, was related to the absence of physical pain at the times of self-injury. The inability to feel either physical or emotional pain would lead people to question their sense of reality. Self-harm, specifically cutting can be used to restore a sense of reality.

\section{Externally motivated functions}

This theme focuses on extrinsic factors that could lead service users to engage in self-harming acts. Implicated in this discussion are feelings of loss of control of external situations. Examples of such situations commonly noted in the transcripts are rape and sexual abuse. Such external events can give rise to a state of high emotional arousal, which can perpetuate people`s need for self-harm.

\section{Punishment: self and others}

Some participants seemed to claim that the behaviour of self-harm is a re-enactment of traumatic experiences of abuse. Individuals who have been subjected to such encounters are more likely to feel evil, bad and guilty. It is this cocktail of emotions participants claimed that sometimes lead to self-injury.

Experiences of sexual abuse often make users feel guilt and sometimes have feelings of lack of control over their lives. They cut to regain control over their bodies (In).

\section{An attempt was made to explain what lack of control means:}

A service user told me that she was overpowered by her abuser when it happened. She now feels compelled to hurt herself. She cut to alleviate the feelings of self-hatred she developed (In).

There was a degree of agreement among some participants that feelings of self-hatred, self-blame and guilt are not uncommon with victims of sexually abuse. Added to this, they acknowledged that individual with these experiences sometimes express feelings of anger and hatred for their perpetrators.

A service user told me that she feels purified the more she cuts. Cutting is sometimes hurting the man, the nasty man (Fg).

Participants believed that practitioners in secure settings may feel obliged to fulfill their professional responsibility. Hence, they are more likely to feel pressurised to offer care to service users when they selfharm.

\section{Influence others}

Some participants of both sets of interviews communicated a shared opinion about self-harm. They claimed that self-injury of any kind, particularly cutting, does have a significant influence on how professionals may react to service users.

Individuals harm to manipulate their care. They cut particularly when the team is reluctant or refusing to meet their needs. When they do it, we rush to talk to them (In).

Self-harm is not just a function of professionals' reluctance or refusal to provide care, it is sometimes, some participants asserted, due to users inability to ask for help.

One service user told me that he does not know how to ask for help. According to him cutting has helped him a lot and he gets nurtured when he cuts (In). 
While most participants recognised that self-harm can be used as a means for "attracting care", other interviewees put forward a contrasting and interesting reason. They stressed that people sometimes harm to shock or drive others away from them.

Some service users liked to be left alone when hurting themselves. They feel disturbed when we intervene. Some sometimes cut deep to push us away (In).

Expression of emotions is a fundamental function of self-harm. In relation to this, participants unanimously refer to this behaviour as a strategy for coping with enormous psychological distress. Although it could sometimes lead to accidental death, some strongly considered it a life-saving exercise rather than a self-destructive one.

\section{Averting death}

A minority of interviewees stated that people who have been exposed to traumatic events, on occasions, do experience mounting internal tensions. This tension was described as a heightened emotional state, composed of an unpleasant mixture of emotions like anxiety, anger, guilt and self-hatred. Additionally, they claimed that self-injury can offer a massive reduction in such tension, which if allowed to grow, could lead to fatal or near fatal outcomes.

A female service user told me that each scar on her body represents a period of time she escaped death (Fg).

Even though self-ham is not an ideal coping approach for nurses, its facilitation of emotional release can in some instances prevent or at least delay suicide attempts. Such affect regulatory and survival functions were believed to act as reinforcers for this behaviour. There are factors within secure clinical settings that can also contribute to and maintain service users ' distress and subsequent self-harming behaviours.

\section{Detention and institutional factors}

It was indicated at interviews that some service users start to hurt themselves when admitted to secure environments. This was attributed to feelings of disempowerment, powerlessness, not being listened to and being confined in a controlled setting.

\section{Controlled environment}

Attempts were made by participants to clarify the meaning of a controlled environment. They referred to it as a setting in which people are being locked, closely observed, monitored and engaged in treatment. Some thought of it as a setting that enables service users to reminisce about abuses they suffered during childhood.

Individuals with at risk of killing themselves are usually "specialled". We even enter the bathrooms when they are taking care of their needs. This means of control reminds them of their past trauma of abuse ( Fg).

Being subjected to such forms of monitoring would be distressing to anyone. It would be even more distressing or traumatic for someone who has been exposed to abusive encounters. However, some participants highlighted that it is the confinement, not the monitoring that plays a major role in generating distress.

Users mainly lacerate their bodies to cope with the feelings of being locked up and the many rigid rules that go with it (Fg).

A participant provided examples of the possible effects of rules when explaining what a controlled environment means.

It is one that would not help people to grow because they just have to listen and do exactly what they are told. This manner of responding relates to our limited knowledge about self-harm (In).

Participant later articulated the meaning of "not helping people to grow" and its impact:

Users are not involved in decision making. With time they would lose even the basic skills to deal with personal problems (In).

\section{Depletion of coping skills}

This was reported by just a handful of participants in their efforts to explain its association with self-harm. They claimed that service users who self-harm are generally dependent on them to address their problems.

We tend to make the users hurt themselves by allowing them to be dependent on us. So, some cannot now even cope with the slightest pressure (In).

Self-harming behaviour was attributed to deficits in problemsolving skills. Others related it to the ease of accessing or retrieving information to deal with problems. Participants who believed in the latter claimed that the thoughts of self-harm are more easily accessible and service users are more likely to use the same to address their problems.

We do almost everything for service users. Doing things this way would make them lose their skills. The only skill they are left with is self-harm (Fg).

\section{Stigmatisation}

The perception of how service users are viewed by healthcare professionals can have implications for both care provision and selfharming acts.

On Sunday morning shift, a user hurt herself three times. Consequently, the ward became chaotic. Staff blamed her for this and ignored her for most part of the shift. She felt stigmatised and guilty for the chaos and harmed in the afternoon (Fg).

People can hurt themselves if they feel stigmatised (enact stigma), and may be subjected to discriminatory practices if stigmatised or labelled by practitioners (express stigma). Participants claimed that labels would generate feelings of humiliation and loss of hope, which, in turn, would result in more self-harming acts.

One thing that promotes self-harm is when they feel stigmatised because of the names or labels we give them. We certainly need some training as our knowledge about self-harm is limited (Fg).

\section{Discussion}

Several reasons were cited by participants of this study for service users` self-harming behaviours. Examples of these include influencing others and cleansing. Added to this, issues to do with control, which include feeling out of control, loss of control and the desire to regain control, were frequently discussed during interviews. Clearly, such a multiplicity of reasons is an indication of the complexity of this behaviour. While this is the case, it is necessary to mention that these findings share similarities with previous studies. Taking for example the feelings of being out of control, reports are noted in the literature that children in abusive encounters are believed to have no influence over their abusers, as they can be physically and sexually molested at anytime [19]. It is the opinion of the researcher of this study that exposure to protracted periods of abuse could result in these children becoming accustomed to the experiences of pain. Arguably, when 
children who have had such encounters grow to adulthood and are faced with difficult relationships or stressful situations, they are more likely to expect to be maltreated. While this assertion is attributable to their previous associations of pain with stress, it articulates why adults who have been physically or sexually abused tend to resort to selfharming behaviours.

It is becoming a common knowledge among practitioners that secure environments are hotbeds for self-harming behaviours [3]. Similar outcomes are reflected in this study as participants reported that some users only commence acts of harm when detained in forensic mental health settings. This is certainly not surprising as a number of researchers have consistently cited that being detained and neglected, characteristics which are inherent in secure environments, are significant risk factors for self-harm [16]. It is essential to state that acts of neglect are manifested in these settings because of a social and psychological gap that exists between users and healthcare professionals [18]. Simply, this means that the latter are providers of care and instructions, whilst the former are expected to comply with rules, regulations and care provided with little or no allowance for self-expression. Not having control in such encounters could result in the users (recipients of care) becoming frustrated, anxious, and angry and feeling out of control. Such a cocktail of emotions, which are more likely to be underpinned by strong feelings of being out of control and frightening experiences, would certainly require immediate but safe expression [21]. Self-harm is believed to be the safest channel for venting these emotions, as users may feel uncomfortable to externalise the same against others for fear of repercussions. It certainly plays a part in enabling service users to address their desire for regaining emotional control. Feeling in control of one's emotions would enhance individuals' perceptions of safety.

It could be postulated that the provision of care within an atmosphere of a social and psychological apartheid could be disempowering for service users. This is apparently the case, as it is reiterated by some participants that care provisions in secure care settings are generally not carried out in partnership with service users. This manner of care exchange would certainly over a period of time deplete service users of essential life skills including those of problem solving, leaving selfharm as one of the few options for addressing life difficulties, a view also expressed by participants of this study. While this study partially supports this view, there are indications in the narratives that the use of coping skills is situational and it is only in some instances that "normal skills" are hindered; not applied. Examples of these include situations where individuals feel controlled and being out of control. Thus, exposure to such circumstances in practice, coupled with depletion of skills, may result in users utilising self-harm to regain emotional control. The apparent relationship between self-harm, control and depletion of coping skills is illustrated diagrammatically below in figure 1. This will enables readers to develop an overview of the context in which self-harm tends to occur in secure settings. The direction of arrows in the figure shows the sequence of events which relate to aspects of control that may lead to self-harming behaviours. Detention and its associated environmental controls, rigid rules and negative attitudes can generate feelings of powerlessness, frustration and anger that may trigger self-injury. This in turn may lead to higher levels of control, further increasing levels of frustration, leaving service users with little motivation to deal with the reasons for self-injury (Figure 1).

The desire to achieve emotional control is not the only motive for self-harm, individuals also tend to engage in it to attain a state of emotional calmness when distressed. A number of researchers have consistently cited that coping with unbearable feelings is one of the most common motives for self-harming behaviours [15]. This manner of

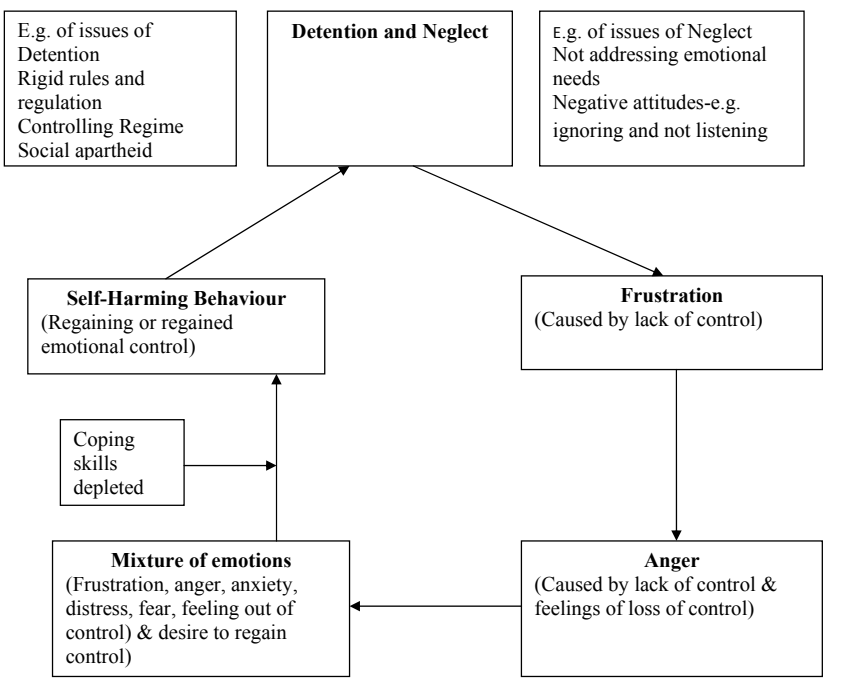

Figure 1: Self-Harm Explanatory Model (SEM).

dealing with distress is utilised by people for a range of reasons. Perhaps, the most frequently reported purpose, as asserted by Snow [8], is regulation of distress, a finding consistent with the views of participants of this study. Some participants claimed that users in their clinical areas usually bottle-up angry emotions that are by and large generated by the overpowering and malevolent nature of secure environments $[11,20]$. In these circumstances, individuals will be become stressed and such stress can ultimately lead to anger and frustration if the problems are not addressed. In such a heightened emotional state, customary ways of coping, which include social interaction and activities, are usually ineffective in restoring emotional calmness. However, there appears to be a growing consensus among many researchers that self-harm is an effective means for minimising tension to bearable levels, an opinion also reflected in this inquiry [10]. Such usage could negatively reinforce the behaviour when people in heightened emotional states achieve calmness or reduction in tension. Arguably, self-harm can be considered a learned behaviour. This means that people who have maintained a calm emotional state by hurting themselves may continue to do so whenever they encounter unbearable experiences, since unbearable encounters are unpleasant and uncomfortable places to be.

Several studies have shown that negative reinforcement, reduction in tension, maintains self-harming behaviour [12,23]. It is therefore not surprising to note an increase in repetition of this behaviour in clinical practice [24]. Notably, such an increase seems to indicate persistent and re-occurring distress in those repeating the behaviour. Taking this argument of emotional relief further, participants of this study seem to believe that people who have been exposed to distressing events, on occasions, do experience mounting internal tensions with overwhelming thoughts of wanting to kill themselves. Self-harm is used in these extreme cases to facilitate emotional release with a view to relieving affected individuals from emotional distress. Simply, it serves an alternative to suicide in instances where people feel unsafe and being out of control of their emotions. It is therefore a coping strategy considered by participants to offer users a sense of emotional control.

\section{Strengths and Limitations}

This study focuses solely on psychiatric nurses ' explanations of selfharming behaviours in secure environments. Thus, further research is needed to explore service users ' as well as and psychiatric nurses' perspectives or explanations of self-harm within these settings. Doing 
so would enhance insight into this behaviour. The study was carried in a single Trust and utilised a purposive sample of psychiatric nurses to capture their views of self-harm. It is important to acknowledge that no two secure environments are the same, and this is also the case of the nurses working within them. Hence, the findings of this study are not generalisable across all secure settings in the United Kingdom. However, they are transferable to other secure environments as they provide insights for understanding psychiatric nurses' meanings and explanations of self-harming behaviours. The study demonstrates that individual and focus group interviews are effective methods for researching perceptions of service users` motives for self-harm.

\section{Conclusion}

A wide range of reasons for self-harm was reported by participants of the current inquiry. It is claimed that self-harm has the function of expressing anger, distress and protest. It is also believed to be a means individuals use to gain a sense of control over some aspects of their lives. Thus, preventing users from harming themselves, could result in their anger and wish for control to be expressed outwardly at healthcare professionals, objects and other members of practice settings. Acknowledging this, the provision of alternative means for emotional expression should be part and parcel of prevention strategies.

It is illustrated in the results of this study that self-harm and suicide or attempted suicides are separate acts, with different behavioural intentions. According to some participants, self-harm is sometimes undertaken by service users to avert suicidal feelings. For these service users, they may harm themselves whenever they are having tormenting thoughts of ending their lives. While this provides an explanation for the increased risk of suicide for individuals who repeatedly self-harm, it is critical to state that this behaviour only temporarily alleviates people's distress. Taking this into account, the occurrence of self-harm should be considered a call for intense engagement to address the underlying motives for the behaviour.

However, participants claimed that their knowledge about selfharm is limited. This suggests that they may experience difficulties in offering explanations of service users' self-harming behaviours. From a clinical perspective, this is of grave concern since service users who self-harm are often confused about their behaviours, and therefore rely on healthcare professionals, including psychiatric nurses to understand what is happening to them. Psychiatric nurses who fail to understand and explain self-harming behaviours are unlikely to establish the collaborative or therapeutic alliance necessary to positively influence behaviour change. Therefore regular training and support should be provided for psychiatric nurses in secure environments in order to broaden their understanding of self-harm and its underlying motives. The findings of the present study indicate that repeated self-harming behaviours in the face of substantial effort to prevent the same can cause psychiatric nurses to feel frustrated, anxious and angry with possible thoughts of being a failure. The next paper will report on the impact of self-harming behaviours on healthcare professionals.

\section{Acknowledgements}

The authors gratefully acknowledge the psychiatric nurses of the study site who offered their experiences of working with people who self-harm. This research received no specific grant from any funding agency.

\section{References}

1. Cooper J, Kapur N, Webb R, Lawlor M, Guthrie E, et al. (2005) Suicide after deliberate self-harm: a 4-year cohort study. Am J Psychiatry 162: 297-303.

2. Hawton K, Bale L, Casey D, Shepherd A, Simkin S, et al. (2006) Monitoring deliberate self-harm presentations to general hospitals. Crisis 27: 157-163.
3. Gough K (2005) Guidelines for managing self-harm in a forensic setting. British Journal of Forensic Practice 7: 10-14.

4. Duperouzel H, Fish R (2010) Hurting No-One Else's Body but Your Own: People with Intellectual Disability Who Self Injure in a Forensic Service. Journal of Applied Research in Intellectual Disabilities 23: 606-615.

5. Patterson P, Whittington R, Bogg J (2007) Measuring nurse attitudes towards deliberate self-harm: the Self-Harm Antipathy Scale (SHAS). J Psychiatr Ment Health Nurs 14: 438-445

6. Ystgaard M, Arensman E, Hawton K, Madge N, van Heeringen K, et al. (2009) Deliberate self-harm in adolescents: comparison between those who receive help following self-harm and those who do not. J Adolesc 32: 875-891.

7. Williams JM (1997) Cry of pain: understanding suicide and self-harm. Penguin Books, Harmondsworth.

8. Snow L (2002) Prisoners' motives for self-injury and attempted suicide. British Journal of Forensic Practice 4: 18-29.

9. Klonsky ED (2007) The functions of deliberate self-injury: a review of the evidence. Clin Psychol Rev 27: 226-239.

10. Long M, Jenkins M (2010) Counsellors' perspectives on self-harm and the role of the therapeutic relationship for working with clients who self-harm. Counselling and Psychotherapy Research 10: 192-200.

11. Favazza AR (1996) Bodies under Siege: Self-mutilation and Body Modification in Culture and Psychiatry. (2nd edn), John Hopkins university press, Baltimore.

12. James M, Warner S (2005) Coping with their lives - women, learning disabilities, self-harm and the secure unit: a Q-methodological study. British Journal of Learning Disabilities 33: 120-127.

13. Pembroke L (2006) Limiting self harm. Emerg Nurse 14: 8-10.

14. Babiker G, Arnold L (1997) The Language of Injury: comprehending SelfMutilation. British Psychological Society, Leicester

15. Hawton K, Bergen H, Casey D, Simkin S, Palmer B, et al. (2007) Self-harm in England: a tale of three cities. Multicentre study of self-harm. Soc Psychiatry Psychiatr Epidemiol 42: 513-521.

16. Tantam D, Huband H. (2009) Understanding repeated self-injury: a multidisciplinary approach. Palgrave Macmillan, UK

17. Low G, Jones D, MacLeod A, Power M, Duggan C (2000) Childhood trauma dissociation and self-harming behaviour: a pilot study. Br J Med Psychol 73 269-278.

18. Goffman E. (1961) Asylums: assays on the social situations of mental patients and other inmates. Pilican, Middlesex

19. Shepperd C, McAllister M (2003) CARE: A framework for responding therapeutically to the client who self-harms. J Psychiatr Ment Health Nurs 10 442-447.

20. Brown J, Beail N (2009) Self-harm people with learning disabilities living in secure service provision: a qualitative exploration. Journal of Applied Research in Intellectual Disabilities 503-513.

21. Hale R (1999) Who is killing whom? The dynamics of self-harm. conference presentation. The Oxford University counselling service annual Conference. St Catherine's College, Oxford.

22. Harker-Longton W, Fish R (2002) 'Cutting doesn`t make you die'one woman's views on the treatment of her self-injurious behaviour. Journal of Intellectual Disabilities 6: 137-151.

23. Walsh BW, Rose PM (1988) Self-mutilation: theory, research and treatment Guildford press, London.

24. Owens D, Horrocks J, House A (2002) Fatal and non-fatal repetition of selfharm. Systematic review. Br J Psychiatry 181: 193-199.

25. Cook SH, Clancy C, Sanderson S (2004) Self-harm and suicide: care interventions and policy. Nurs Stand 18: 43-52.

26. Suyemoto KL (1998) The functions of self-mutilation. Clin Psychol Rev 18: 531 554.

27. Smith JA. (2005) Semi-structured interviewing and qualitative analysis. In Smith JA, Harre R, Van Langenhove L. (eds) Rethinking methods in psychology. Sage, London. 
Citation: Sandy PT (2012) Psychiatric Nurses Explanations of Self-Harming Behaviour in Secure Forensic Settings: A Multi-Method Phenomenological Investigation. J Nurs Care 1:120. doi:10.4172/2167-1168.1000120

Page 7 of 7

28. Langdridge D. (2007) Phenomenological psychology: theory, research and method. Pearson, England

29. Gadamer H. (1996) Truth and method. Sheed and Ward, London.
30. Smith JA, Flowers P, Larkin M. (2009) Interpretive phenomenological analysis: theory, method and research. Sage, London.

31. Smith JA, Osborn M. (2003) Interpretative phenomenological analysis. In. JA Smith. (Ed), Qualitative psychology. Sage, London. 